\title{
LA RIVOLUZIONE DELLA CRIO-MICROSCOPIA ELETTRONICA NELLO STUDIO STRUTTURALE DI PROTEINE E ACIDI NUCLEICI
}

\author{
Nota del s.c. MARTINO BOLOGNESI (*)
}

(Adunanza del 16 novembre 2017)

SunTO. - La Biologia Strutturale si è occupata negli ultimi 50 anni di studiare i dettagli fini delle strutture molecolari subcellulari (es. proteine o acidi nucleici). In tempi recenti, lo sviluppo della crio-microscopia elettronica in singola particella ha portato ad una nuova rivoluzione in questo campo di studio, in precedenza dominio della cristallografia a raggi $\mathrm{X}$ e della risonanza magnetica nucleare. Oggi è possibile studiare direttamente le strutture dei grandi aggregati macro-molecolari (es. il ribosoma) con un potere risolutivo che, nei casi migliori, giunge a $2 \AA$, permettendo di distinguere $i$ dettagli fini di aminoacidi e nucleotidi.

ABSTRACT. - Observing the fine details of molecular structures (e.g. in proteins and in nucleic acids) has been a central part of Structural Biology over the past 50 years. The recent advent of single particle cryo-electron microscopy brought a revolution in this field, that previously relied on X-ray crystallography and nuclear magnetic resonance. It is now possible to explore the structures of large subcellular assemblies, such as the ribosome, resolving details on the scale of amino acids and nucleotides, in favorable cases reaching the $2 \AA$ resolution level.

La comprensione delle basi molecolari dei processi biologici si fonda sulla conoscenza delle modalità di interazione tra atomi/aminoacidi/nucleotidi e altre molecole diverse in uno specifico contesto cellulare o macromolecolare. Interessa anche come si formino/rompano

(*) Dipartimento di Bioscienze, Università degli Studi di Milano, Italy.

E-mail: martino.bolognesi@unimi.it 
legami covalenti, come siano organizzate le strutture tridimensionali (3D) delle proteine, degli acidi nucleici e di altre (macro)molecole biologiche. Sono queste le sfide scientifiche della Biologia Strutturale, che nel corso degli ultimi decenni ha visto riconosciuti diversi premi Nobel nei campi della cristallografia a raggi-X e della risonanza magnetica nucleare (NMR) (per una panoramica dei risultati prodotti da questi due metodi di studio si consulti il data-base Protein Data Bank: www.rcsb.org/pdb). Cristallografia e NMR hanno fornito informazioni preziose, ad esempio per lo sviluppo di nuovi farmaci, producendo più di 140.000 strutture 3D di proteine, o loro complessi, a risoluzione atomica.

L'analisi di corpuscoli sub-cellulari, quindi "oggetti" molto piccoli, può essere svolta con un microscopio ottico. Tuttavia, la risoluzione spaziale di questo strumento cade in difetto quando il campione/dettaglio da analizzare sia di dimensioni inferiori a quelle della lunghezza d'onda della luce visibile (orientativamente minore di $300 \mathrm{~nm}$ ). Grazie agli studi di de Broglie, nei primi decenni del XX secolo, sappiamo che particelle cariche in movimento godono delle proprietà ondulatorie caratteristiche di un fascio di luce. Essendo gli elettroni particelle elementari dotate di una carica negativa, essi possono essere diretti sul campione, realizzando uno strumento che ha le caratteristiche di un vero e proprio microscopio, ma con capacità risolutive molto maggiori del microscopio ottico. In accordo con questa osservazione, negli anni '30 del '900, Ernst Ruska, riuscì a focalizzare un fascio di elettroni attraverso il campo magnetico generato da un solenoide, realizzando di fatto la prima "lente per elettroni", e costruì il primo prototipo di microscopio elettronico. Tale scoperta fu riconosciuta con il Nobel per la Fisica nel 1986 e con l'inizio della diffusione di questi strumenti.

L'evoluzione tecnologica dei microscopi elettronici ha reso disponibile un potentissimo e nuovo metodo di indagine in Biologia, dapprima, e in tempi recenti anche nell'ambito della Biologia Strutturale. Gli elettroni infatti interagiscono con gli atomi in maniera più efficiente dei raggi $\mathrm{X}$, possono essere focalizzati con lenti elettromagnetiche, presentano lunghezze d'onda molto brevi (dell'ordine di qualche centesimo di $\AA$ ) dal punto di vista ondulatorio, e permettono di esaminare i campioni in soluzione o addirittura in cellula. In linea di principio, la microscopia elettronica può quindi permettere di risolvere atomi di carbonio legati covalentemente in una proteina o in una molecola di DNA, le cui distanze cadono nell'intervallo $1.2-1.6 \AA$. 
Tuttavia, l'applicazione della microscopia elettronica a campioni biologici non è così immediata come poterbbe apparire. Infatti, L'energia degli elettroni accelerati nel microscopio viene trasferita al materiale organico in esame e lo danneggia sensibilmente. Inoltre, per consentire il percorso ottico degli elettroni attarverso lo strumento, la camera interna del microscopio deve essere mantenuta ad elevati livelli di vuoto $\left(<10^{-7} \mathrm{~Pa}\right)$, notoriamente poco compatibili col mantenimento di condizioni native per un campione biologico.

Per ovviare a questi inconvenienti si è sviluppata la cryo-electron microscopy (Cryo-EM), metodo secondo cui il campione biologico in soluzione viene rapidamente congelato per immersione in un agente criogenico (ad esempio, etano o azoto liquidi), tramite un processo definito come "vitrificazione". A temperature dell'azoto liquido il danno da radiazione è ridotto, in quanto i radicai liberi creati dall'interazione del campione con gli elettroni hanno ridotte possibilità di diffusione, quindi di danno alle molecole presenti nel campione.

L'esperienza maturata nelle prime applicazioni Cryo-EM, tuttavia, ha indicato che il crio-congelamento da solo risolve in maniera sufficiente il problema del danno indotto dalla radiazione elettronica. Una vera "rivoluzione" in questa direzione si è riscontrata a partire dal 2012, con lo sviluppo di una nuova generazione di dispositivi, definiti Direct Electron Detectors (DED), caratterizzati da una elevata efficienza nella registrazione del segnale. A differenza di altri dispositivi (lastre fotografiche o CCD), in un DED l'elettrone viene registrato direttamente dal sensore costituito da ossidi metallici semiconduttori (tecnologia CMOS, che non richiede conversioni del segnale elettronico in radiazione del visibile); inoltre, il sensore DED presenta una velocità di acquisizione elevata, che permette di registrare centinaia di immagini/secondo. Per acquisire un segnale trattabile, un DED richiede che il campione venga irradiato con dosi bassissime di elettroni; pertanto si affianca in mnaiera sinergistica al processo di vitrificazione come espediente attivo nella riduzione del danno da radiazione al campione.

In un esperimento di crio-microscopia elettronica a singola particella (spCryo-EM) per studiare la struttura 3D di un complesso proteico (vitrificato in soluzione), migliaia d'immagini, dette micrographs, vengono registrate nello strumento (1-5 Tbytes di dati per esperimento). Ogni micrograph contiene centinaia di macromolecole, dette single particles, distribuite nella soluzione vitrificata. L'insieme complessivo dei dati acquisiti raccoglie centinaia di migliaia di campionature di sin- 
gole molecole che vengono riconsosciute singolarmente e combinate secondo orientazione e conformazione assunta, al fine di sommare $\mathrm{i}$ loro segnali e minimizzare il rumore di fondo. Da questa elaborazione si passa poi ad una procedura computazionale più impegnativa, per teoria, carico di calcolo e tempi di esecuzione. Questa fase permette di ricostruire la struttura 3D del complesso proteico (la single particle) in esame; con gli strumenti più avanzati disponibili, la risoluzione raggiungibile si attesta tra 3 e $2 \AA$, a seconda delle qualità del campione, e in casi favorevoli l'analisi può richiedere 2-3 settimane.

L'impatto della spCryo-EM sulla Biologia Strutturale, e sul mondo della Biologia in generale, è stato visibile e sostanziale a partire dal 2015, con sviluppi e installazioni molto importanti nei principali laboratori di ricerca di tutto il mondo. In particolare, un intero campo di studio (quello delle proteine di membrana, che sono i bersagli di almeno il 30\% dei farmaci maggiormente attivi) ha visto una progressione insperata e promettente, sia per le potenzialità applicative, sia per la rapidità delle risposte sperimentali. Non è un caso che il Premio Nobel per la Chimica 2017 sia stato conferito a Jacques Dubochet (Università di Losanna), Joachim Frank (Columbia University, New York) e Richard Henderson (MRC Laboratory of Molecular Biology, Cambridge, UK) "for developing cryo-electron microscopy for the highresolution structure determination of biomolecules in solution". 